\title{
Avaliação retrospectiva por meio do questionário SF-36 de pacientes submetidos à estabilização dinâmica pedicular para o tratamento de doenças degenerativas lombares
}

Retrospective evaluation by means of SF-36 questionnaires of patients submitted to pedicular dynamic stabilization for degenerative lumbar diseases treatment

Evaluación retrospectiva empleando el cuestionario SF-36 en pacientes sometidos a estabilización dinámica pedicular para el tratamiento de la enfermedad degenerativa lumbar

\author{
Leonardo Fonseca Rodrigues ${ }^{1}$ \\ Paula Voloch ${ }^{2}$ \\ Sérgio Gurgel ${ }^{3}$ \\ Flavio Cavallari ${ }^{4}$
}

\section{RESUMO}

Introdução: a estabilização dinâmica pedicular tem sido utilizada desde os anos 1990 para o tratamento da doença lombar degenerativa como uma alternativa aos métodos de fusão. Devido às suas características de não-fusão, estes implantes tendem a reduzir a carga nos discos intervertebrais adjacentes e na coluna posterior, com o objetivo de não prejudicar a biomecânica da coluna, diminuindo assim a chance de degeneração do disco adjacente. Objetivo: O objetivo deste estudo retrospectivo foi avaliar, por meio do questionário SF-36, os resultados dos pacientes que se submeteram à estabilização dinâmica pedicular para o tratamento da patologia degenerativa lombar. Métodos: foram avaliados 31 pacientes que se

\begin{abstract}
Introduction: pedicular dynamic stabilization has been used, since the 1990 's, for treatment of degenerative lumbar diseases as an alternative to fusion methods. Due to its nonfusion features, these implants tend to reduce the load to the adjacent intervertebral discs and in the posterior column, aiming at not affecting the spine biomechanics, thus decreasing the chance of adjacent disc degeneration. Objective: the purpose of this retrospective study was to evaluate the quality of life, by means of SF-36 questionnaires, of patients who underwent pedicular dynamic stabilization for treatment of degenerative lumbar pathology. Methods: thirty-one patients who were submitted to pedicular dynamic
\end{abstract}

\section{RESUMEN}

Introducción: la estabilización dinámica pedicular ha sido empleada desde los años 1990 en el tratamiento de la enfermedad degenerativa lumbar y como una alternativa a los métodos de fusión. Debido a sus características de no requerir fusión, estos implantes tiendes a reducir la carga sobre los disco intervertebrales adyacentes y sobre la columna posterior, con el propósito de no afectar la biomecánica vertebral. Por lo tanto, disminuye la degeneración de discos adyacentes. Objetivo: esto estudio retrospectivo tiene la finalidad de evaluar los resultados obtenidos al emplear el cuestionario SF-36, en pacientes que han presentado estabilización dinámica pedicular durante el tratamiento de la patología degenerativa da columna

\footnotetext{
Trabalho realizado nos Centro Ortopedico da Barra - Barra da Tijuca - Rio de Janeiro (RJ), Brasil e Hospital São Vicente de Paulo -Tijuca - Rio de Janeiro (RJ), Brasil.

${ }^{1}$ Médico do Grupo de Cirurgia da Coluna Vertebral do Serviço de Ortopedia e Traumatologia do Hospital Geral do Andaraí - HGA - Rio de Janeiro (RJ), Brasil; Médico do Serviço de Ortopedia e Traumatologia do Hospital São Vicente de Paulo - HSVP - Rio de Janeiro (RJ), Brasil.

${ }^{2}$ Mestre; Médica do Grupo de Cirurgia da Coluna Vertebral do Serviço de Ortopedia e Traumatologia do Hospital Geral do Andaraí - HGA - Rio de Janeiro (RJ), Brasil.

${ }^{3}$ Médico do Serviço de Ortopedia do Hospital Universitário Clementino Fraga Filho da Universidade Federal do Rio de Janeiro - UFRJ - Rio de Janeiro (RJ), Brasil. ${ }^{4}$ Médico chefe do Grupo de Cirurgia da Coluna Vertebral do Serviço de Ortopedia e Traumatologia do Hospital Geral do Andaraí - HGA - Médico do Serviço de Ortopedia e Traumatologia do Hospital São Vicente de Paulo - HSVP - Rio de Janeiro (RJ), Brasil.
} 
submeteram à estabilização dinâmica pedicular, de abril de 2004 a junho de 2008, por meio do questionário de qualidade de vida SF-36. Resultados: o SF-36 mostrou um índice de melhora na qualidade de vida desses pacientes durante o período de acompanhamento, variando de $33,15 \%$ no pré-operatório, para $75,99 \%$ no pósoperatório, correspondente a uma diferença de $129 \%$ no resultado final, que é significativo estatisticamente de acordo com o teste $t$ de student $(\mathrm{p}<0,0001)$. Conclusões: os pacientes submetidos à estabilização pedicular dinâmica em nossa série mostraram uma melhoria na qualidade de vida, comparando-se os questionários SF-36 pré e pós-operatório em 29,5 meses de acompanhamento. stabilization were evaluated, from April 2004 to June 2008, using the $S F-36$ quality of life questionnaire. Results: the SF-36 index showed an improvement in the quality of life of these patients during the follow-up period, ranging from $33.15 \%$ preoperatively to $75.99 \%$ in postoperatively, showing a difference of $129 \%$ in the result, which is statistically significant according to the Student's $t$ test $(p<0,0001)$. Conclusions: patients submitted to dynamic pedicular stabilization in our series showed an improvement in quality of life when comparing the pre and postoperative SF-36 questionnaires during the 29.5 months follow-up period. lumbar. Métodos: fueron evaluados 31 pacientes que presentaban estabilización dinámica pedicular desde abril de 2004 a junio de 2008, empleando el cuestionario de calidad de vida SF-

36. Resultados: durante el periodo de seguimiento, el indice SF-36 mostró un mejoramiento en la calidad de vida de los pacientes, con un rango del $33.15 \%$ en el preoperatorio a $75.99 \%$ en el postoperatorio, mostrando una diferencia de $129 \%$, lo cual es una diferencia significativa utilizando la estadística teste t de Student $(p<0.0001)$. Conclusiones: los pacientes estudiados que fueron sometidos a la estabilización dinámica pedicular mostraron un mejoramiento en la calidad de vida, se han comparado los cuestionarios SF-36 en pre y post operación durante 29.5 meses de seguimiento.

DESCRIPTORES: Dolor de la

\section{región lumbar; Estabilización/
métodos; Inestabilidad de la
articulación; Cuestionarios \\ región lumbar; Estabilización/
métodos; Inestabilidad de la
articulación; Cuestionarios \\ región lumbar; Estabilización/
métodos; Inestabilidad de la
articulación; Cuestionarios}

KEYWORDS: Low back pain; Stabilization/methods; Joint instability; Questionnaires Questionários

Estabilização/métodos; Instabilidade articular;

\section{INTRODUÇÃO}

O sucesso do tratamento cirúrgico das patologias da coluna lombar está relacionado ao correto diagnóstico da origem da dor. Contudo, o sintoma doloroso, por ser muitas vezes subjetivo, é extremamente complexo. Portanto, a definição de sua origem é fundamental para a escolha da melhor estratégia cirúrgica.

A estabilidade lombar, definida por White e Panjabi ${ }^{1}$ em 1990, pode ser resumida como "a capacidade da coluna vertebral, sob carga fisiológica nos limites padrões do deslocamento, de não causar dano ou irritação na medula, cauda eqüina ou raízes nervosas e, também, prevenir deformidade incapacitante, dor ou mudanças estruturais".

A importância da instabilidade segmentar lombar foi citada inicialmente por Kruton², em 1944. Morgan e $\mathrm{Krug}^{3}$ relataram a instabilidade como causa primária de lombalgia em 1957. O processo degenerativo lombar começou a ser mais bem compreendido com os estudos de Kirkaldy-Willis et al. ${ }^{4}$, em 1978, e a evolução da patologia através da cascata degenerativa, descrita posteriormente por Kirkaldy-Willis e Farfan ${ }^{5}$, em 1982.

As técnicas de tratamento cirúrgico da lombociatalgia evoluíram em função dos resultados insatisfatórios obtidos após as discectomias convencionais a médio e longo prazo. A estabilização do segmento lombar degenerado através da artrodese passou a ser uma opção terapêutica, tornando-se um procedimento mais frequente. O principal objetivo para indicação da estabilização lombar é tratar a dor de origem facetária ou discal, cuja característica é a manifestação sob carga ${ }^{6}$.

A coluna lombar não é um sistema articular único. Ela é dividida em unidades funcionais, que são as unidade móveis da coluna vertebral, composta por duas vértebras, duas articulações facetarias e um disco (três articulações) ${ }^{7}$. Os corpos vertebrais se alinham em diferentes ângulos com as vértebras adjacentes, dependendo da localização desta unidade funcional ${ }^{8}$.

A biomecânica da coluna vertebral não é simples, pois envolve movimentos complexos de flexão, extensão, inclinação lateral e rotação, bem como a combinação de todos estes movimentos. Seu centro de rotação não é estático como no quadril. Existe uma mudança deste centro conforme a combinação dos movimentos, o que determina diferentes pontos de carga axial na mesma unidade espinhal funcional que incidem sob o disco intervertebral e as articulações facetárias ${ }^{9}$.

São estas peculiaridades inerentes à coluna lombar que tornam difícil a escolha do tratamento diante das opções de técnicas cirúrgicas disponíveis. A indicação mais comum para o tratamento cirúrgico da patologia degenerativa lombar é a artrodese ou fusão, que apresenta bons resultados em aproximadamente $70 \%$ dos casos, segundo Christensen, em 
uma avaliação a longo prazo ${ }^{6}$. Porém, complicações em médio prazo das fusões lombares costumam ocorrer por sobrecarga nos níveis vertebrais adjacentes, determinando degeneração discal adjacente (DDA). Segundo Rham e Hal ${ }^{10}$, a DDA ocorre, em média, em $30 \%$ dos casos, após cinco anos. A hipermobilidade articular do nível adjacente foi registrada por Luk et al. ${ }^{11}$ em 50\% no segmento acima, dos quais 30\% apresentavam também estenose do canal lombar. Existe, ainda, a pseudoartrose como fator de complicação pós-cirúrgica para comprometer o resultado, segundo Kornblum ${ }^{12}$. A consolidação óssea é fundamental para o sucesso cirúrgico da artrodese lombar, conforme relatado por Butterman et al. ${ }^{13}$ em estudo feito em 1998. Em contrapartida, em 2002, Mulholland e Sengupta observaram que o sucesso da artrodese não representa necessariamente sucesso clínico no tratamento da dor ${ }^{14}$.

Com essas evidências, as técnicas de não-fusão surgem com a proposta de evitar a degeneração do disco adjacente, não causar hipermobilidade articular acima da fusão e preservar o arco articular de movimento da coluna lombar. Este fenômeno foi demonstrado em 1996, por Rham e $\mathrm{Hal}^{10}$, nos pacientes que evoluíram com pseudoartrose onde havia micromovimentos nas articulações facetárias. Os autores descrevem a pseudoartrose como "fator de proteção para o desenvolvimento da degeneração do segmento adjacente", conclusão também observada por Ghiselli et al. ${ }^{15} \mathrm{em}$ estudo realizado em 2004.

Os procedimentos de não-fusão em desenvolvimento atualmente são as artroplastias com substituição total dos discos sem abordagem para as articulações facetárias, e as técnicas de estabilização dinâmica, transpedicular ou interespinhosa. Tais sistemas visam à preservação do movimento articular vertebral com maior ou menor restrição do arco de movimento.

\section{OBJETIVO}

O objetivo deste trabalho foi avaliar retrospectivamente, por meio do questionário de qualidade de vida SF36, o resultado obtido com tratamento cirúrgico das patologias degenerativas lombares através de um sistema de estabilização dinâmica com fixação posterior usando parafusos pediculares revestidos com hidroxiapatita.

\section{MÉTODOS}

Foram avaliados 31 pacientes submetidos à fixação dinâmica pedicular no período de abril de 2004 a junho de 2008 e que aceitaram responder ao questionário de qualidade de vida SF-36. Os questionários relacionados aos períodos pré e pós-operatórios foram respondidos pelos pacientes em dezembro de 2008 (avaliação retrospectiva), acompanhados por um observador externo (Tabela 1).

Todos esses pacientes apresentavam, no pré-operatório, lombalgia mecânica incapacitante, refratários a pelo menos 12 meses de tratamento conservador, incluindo uso de anti-inflamatórios, fisioterapia, perda ponderal, restrição de atividade física e infiltrações.
Os critérios de exclusão para este tipo de tratamento foram: escoliose, tumores, fraturas, infecções, doenças inflamatórias, tabagismo, alcoolismo, osteoporose e necessidade de instrumentação acima de três níveis.

Todos os pacientes foram avaliados no pré-operatório por meio de radiografias simples da coluna lombo-sacra em ortostase, nas incidências anteroposterior (AP) e perfil (P), e imagens de ressonância magnética (IRM), para avaliação da patologia discal e dos níveis a serem abordados.

$\mathrm{O}$ diagnóstico mais frequente foi doença degenerativa discal (DDD), presente em 24 pacientes. Os outros diagnósticos foram: espondilolistese degenerativa em um paciente, degeneração discal adjacente (DDA) após artrodese em um paciente, hérnia discal recorrente após microdiscectomia em quatro pacientes, e ruptura interna discal em um paciente (Figura 1).

O sistema de fixação pedicular utilizado foi o cosmicUlrich, desenvolvido por Strempel et al. ${ }^{16}$ (Figura 2). A principal característica do parafuso é o arco de movimento de $15^{\circ} \mathrm{em}$ sua cabeça, permitindo movimento no sentido crânio-caudal num sistema semiconstricto. Tal mobilidade deixa a articulação facetária livre para micromovimentos. Neste sistema, é possível realizar distração dos segmentos estabilizados através das hastes de conexão. Os parafusos são revestidos com hidroxiapatita para promover osteointegração da prótese ao corpo vertebral, uma vez que toda a fixação depende da qualidade óssea ${ }^{16}$. A inserção do parafuso é feita pela técnica transpedicular, sendo que os parafusos devem convergir em $30^{\circ}$ em direção ao plano sagital, sendo os mais longos possíveis, e a cabeça dos parafusos deve ficar mais próxima do osso, para diminuir as forças de cisalhamento (Figura 3).

A média de idade dos pacientes incluídos no estudo foi de 48,54 anos (com variação entre 25 e 80 anos), sendo 23 mulheres e 8 homens. A estabilização dinâmica foi feita em 1 nível em 13 pacientes, sendo 4 no nível L4-L5 e 9 no nível L5-S1 (Figuras 4 e 5), em 2 níveis em 12 pacientes, todos nos níveis L4-L5 e L5-S1 (Figura 3) e em 3 níveis em 6 pacientes, todos nos níveis L3-L4, L4-L5 e L5-S1 (Figura 6). Cinco pacientes apresentavam comorbidades em tratamento na época do procedimento cirúrgico: um era portador do vírus HIV em tratamento medicamentoso, sem apresentar repercussão clínica no momento da cirurgia; dois pacientes apresentavam hipertensão arterial sistêmica controlada com medicação e dois apresentavam diabetes mellitus compensada com uso de medicação. O período médio de acompanhamento foi de 29,5 meses, com variação entre 7 e 57 meses.

Todos os pacientes foram submetidos ao procedimento cirúrgico com anestesia geral, além de raqui-anestesia com morfina pré-operatória para melhor analgesia no pós-operatório. Os pacientes foram colocados em decúbito ventral sobre o suporte de coluna. $\mathrm{O}$ acesso foi mediano posterior em todos os pacientes, com dissecção da musculatura paravertebral até visualização dos processos transversos, para melhor visualização dos pontos 
TABELA 1 - Resultados do questionário respondido pelos pacientes

\begin{tabular}{|c|c|c|c|c|c|c|c|c|c|c|c|c|c|}
\hline $\begin{array}{l}\frac{0}{\frac{1}{c}} \\
. \frac{0}{0} \\
\frac{0}{2}\end{array}$ & 胥 & 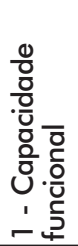 & 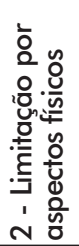 & $\begin{array}{l}\grave{o} \\
\dot{0} \\
\dot{m}\end{array}$ & 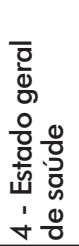 & $\begin{array}{l}\frac{0}{0} \\
\frac{0}{0} \\
\frac{.0}{0} \\
\frac{1}{3} \\
1 \\
\text { in }\end{array}$ & 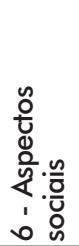 & 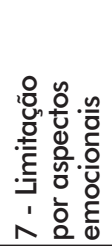 & $\begin{array}{l}\overline{\overline{0}} \\
\frac{1}{\bar{c}} \\
\Phi \\
\varepsilon \\
\frac{0}{0} \\
\frac{0}{0} \\
0 \\
1 \\
\infty\end{array}$ & $\stackrel{.0}{\frac{0}{0}}$ & $\begin{array}{l}\frac{n}{0} \\
\stackrel{2}{c} \\
0 \\
0 \\
\frac{0}{0} \\
\frac{0}{0} \\
\text { Z }\end{array}$ & $\begin{array}{l}\frac{0}{0} \\
\text { 응 }\end{array}$ & 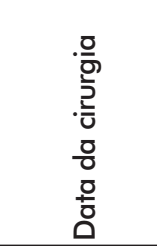 \\
\hline \multirow[t]{2}{*}{$\mathrm{AF}$} & & 5 & 0 & 10 & 52 & 10 & 25 & 33 & 36 & 21,375 & 3 & 45 & $6 / 5 / 2008$ \\
\hline & M & 95 & 100 & 42 & 62 & 60 & 75 & 33 & 80 & 68,375 & & & \\
\hline \multirow[t]{2}{*}{ AFC } & & 15 & 0 & 20 & 25 & 45 & 50 & 0 & 48 & 25,375 & 2 & 43 & $4 / 14 / 2006$ \\
\hline & $\mathrm{F}$ & 70 & 25 & 50 & 62 & 65 & 37,5 & 33 & 60 & 50,3125 & & & \\
\hline \multirow[t]{2}{*}{ AHF } & & 25 & 0 & 50 & 20 & 45 & 25 & 0 & 48 & 26,625 & 3 & 55 & $4 / 22 / 2006$ \\
\hline & M & 90 & 100 & 62 & 87 & 70 & 100 & 100 & 92 & 87,625 & & & \\
\hline \multirow[t]{2}{*}{$A Q C R$} & & 10 & 0 & 50 & 60 & 55 & 37,5 & 0 & 48 & 32,5625 & 2 & 43 & $10 / 3 / 2007$ \\
\hline & $\mathrm{F}$ & 90 & 100 & 72 & 90 & 80 & 75 & 100 & 84 & 86,375 & & & \\
\hline \multirow[t]{2}{*}{ BLGA } & & 90 & 0 & 51 & 92 & 20 & 12,5 & 33 & 36 & 41,8125 & 2 & 25 & $3 / 31 / 2007$ \\
\hline & M & 90 & 0 & 51 & 92 & 20 & 12,5 & 33 & 36 & 41,8125 & & & \\
\hline \multirow[t]{2}{*}{ DFM } & & 35 & 100 & 90 & 77 & 60 & 75 & 100 & 64 & 75,125 & 3 & 78 & $1 / 6 / 2007$ \\
\hline & M & 60 & 100 & 90 & 77 & 60 & 75 & 100 & 68 & 78,75 & & & \\
\hline \multirow[t]{2}{*}{ EGFL } & & 0 & 0 & 50 & 55 & 15 & 37,5 & 0 & 60 & 27,1875 & 1 & 27 & $12 / 18 / 2007$ \\
\hline & $\mathrm{F}$ & 95 & 100 & 62 & 72 & 80 & 87,5 & 100 & 80 & 84,5625 & & & \\
\hline \multirow[t]{2}{*}{ FPR } & & 20 & 0 & 10 & 25 & 15 & 12,5 & 33 & 28 & 17,9375 & 3 & 46 & $10 / 14 / 2006$ \\
\hline & M & 50 & 0 & 42 & 57 & 55 & 37,5 & 33 & 52 & 40,8125 & & & \\
\hline \multirow[t]{2}{*}{ GBCJ } & & 30 & 0 & 31 & 67 & 55 & 12,5 & 0 & 44 & 29,9375 & 1 & 30 & $6 / 10 / 2008$ \\
\hline & M & 55 & 25 & 62 & 67 & 50 & 75 & 0 & 80 & 51,75 & & & \\
\hline \multirow[t]{2}{*}{ GPR } & & 10 & 0 & 20 & 50 & 15 & 25 & 0 & 8 & 16 & 1 & 30 & $4 / 18 / 2006$ \\
\hline & M & 90 & 75 & 62 & 82 & 75 & 100 & 100 & 84 & 83,5 & & & \\
\hline \multirow[t]{2}{*}{ JHS } & & 5 & 0 & 10 & 50 & 0 & 0 & 0 & 8 & 9,125 & 2 & 45 & $4 / 10 / 2008$ \\
\hline & M & 95 & 100 & 90 & 80 & 100 & 100 & 100 & 100 & 95,625 & & & \\
\hline LMGP & & 45 & 100 & 10 & 30 & 55 & 50 & 0 & 56 & 43,25 & 2 & 64 & $3 / 20 / 2007$ \\
\hline & $\mathrm{F}$ & 65 & 0 & 10 & 37 & 35 & 50 & 33 & 68 & 37,25 & & & \\
\hline MATM & & 100 & 100 & 64 & 87 & 65 & 37,5 & 100 & 76 & 78,6875 & 1 & 40 & $10 / 9 / 2007$ \\
\hline & M & 85 & 0 & 20 & 70 & 65 & 37,5 & 100 & 96 & 59,1875 & & & \\
\hline MMR & & 0 & 75 & 50 & 35 & 20 & 12,5 & 67 & 16 & 34,4375 & 1 & 39 & $8 / 14 / 2006$ \\
\hline & M & 100 & 100 & 90 & 100 & 85 & 100 & 100 & 80 & 94,375 & & & \\
\hline MTJBGF & & 90 & 25 & 31 & 67 & 70 & 62,5 & 100 & 76 & 65,1875 & 1 & 57 & $10 / 5 / 2006$ \\
\hline & $\mathrm{F}$ & 24 & 0 & 10 & 52 & 60 & 50 & 0 & 76 & 34 & & & \\
\hline RCPG & & 10 & 0 & 50 & 5 & 5 & 0 & 0 & 4 & 9,25 & 2 & 47 & $4 / 17 / 2007$ \\
\hline & $\mathrm{F}$ & 50 & 100 & 41 & 82 & 85 & 87,5 & 0 & 84 & 66,1875 & & & \\
\hline WH & & 35 & 25 & 41 & 82 & 10 & 37,5 & 33,3 & 32 & 36,975 & 3 & 76 & $11 / 26 / 2006$ \\
\hline & M & 35 & 25 & 64 & 82 & 10 & 12,5 & 0 & 32 & 32,5625 & & & \\
\hline SCFS & & 0 & 0 & 0 & 5 & 0 & 0 & 0 & 0 & 0,625 & 2 & 41 & $6 / 7 / 2005$ \\
\hline & $\mathrm{F}$ & 90 & 100 & 84 & 100 & 70 & 87,5 & 100 & 68 & 87,4375 & & & \\
\hline $\operatorname{cssc}$ & & 35 & 0 & 20 & 40 & 60 & 25 & 0 & 80 & 32,5 & 1 & 48 & $3 / 13 / 2008$ \\
\hline & $\mathrm{F}$ & 100 & 100 & 100 & 100 & 75 & 100 & 100 & 80 & 94,375 & & & \\
\hline MGLR & & 0 & 0 & 32 & 80 & 0 & 0 & 0 & 24 & 17 & 1 & 58 & $7 / 28 / 2005$ \\
\hline & $\mathrm{F}$ & 95 & 100 & 40 & 100 & 95 & 100 & 66,66 & 96 & 86,5825 & & & \\
\hline AGRB & & 10 & 0 & 20 & 60 & 25 & 25 & 100 & 16 & 32 & 1 & 38 & $11 / 26 / 2005$ \\
\hline & $M$ & 100 & 100 & 100 & 72 & 50 & 100 & 100 & 16 & 79,75 & & & \\
\hline JFS & & 0 & 0 & 0 & 15 & 45 & 37,5 & 100 & 28 & 28,1875 & 1 & 51 & $1 / 11 / 2005$ \\
\hline & $\mathrm{F}$ & 100 & 100 & 72 & 27 & 70 & 100 & 100 & 76 & 80,625 & & & \\
\hline WS & & 15 & 0 & 10 & 40 & 30 & 87,5 & 0 & 36 & 27,3125 & 2 & 48 & $12 / 9 / 2004$ \\
\hline & $\mathrm{F}$ & 100 & 100 & 72 & 100 & 80 & 100 & 100 & 72 & 90,5 & & & \\
\hline RCCR & & 15 & 0 & 10 & 5 & 30 & 50 & 0 & 20 & 16,25 & 1 & 42 & $4 / 27 / 2004$ \\
\hline & $\mathrm{F}$ & 100 & 100 & 100 & 100 & 85 & 100 & 100 & 100 & 98,125 & & & \\
\hline CGR & & 45 & 75 & 20 & 15 & 55 & 50 & 100 & 32 & 49 & 1 & 42 & 1/1/2005 \\
\hline & $\mathrm{F}$ & 85 & 100 & 51 & 97 & 75 & 100 & 100 & 76 & 85,5 & & & \\
\hline TLB & & 0 & 0 & 10 & 65 & 35 & 12,5 & 0 & 36 & 19,8125 & 2 & 53 & $3 / 2 / 2006$ \\
\hline & $\mathrm{F}$ & 100 & 100 & 100 & 100 & 100 & 100 & 100 & 100 & 100 & & & \\
\hline VLLC & & 50 & 0 & 20 & 35 & 45 & 87,5 & 0 & 92 & 41,1875 & 2 & 66 & $4 / 25 / 2006$ \\
\hline & $\mathrm{F}$ & 80 & 100 & 50 & 52 & 65 & 100 & 100 & 92 & 79,875 & & & \\
\hline PFSL & & 55 & 25 & 20 & 37 & 55 & 12,5 & 75 & 64 & 42,9375 & 2 & 47 & $9 / 23 / 2004$ \\
\hline & M & 90 & 100 & 72 & 47 & 60 & 100 & 100 & 72 & 80,125 & & & \\
\hline RMCG & & 15 & 0 & 20 & 20 & 60 & 62,5 & 0 & 36 & 26,6875 & 2 & 34 & $3 / 22 / 2005$ \\
\hline & $\mathrm{F}$ & 100 & 100 & 100 & 100 & 60 & 100 & 100 & 80 & 92,5 & & & \\
\hline VAJ & & 55 & 50 & 51 & 87 & 50 & 100 & 0 & 64 & 57,125 & 1 & 80 & $10 / 7 / 2006$ \\
\hline & $\mathrm{F}$ & 55 & 25 & 80 & 87 & 60 & 100 & 0 & 68 & 59,375 & & & \\
\hline JMJA & & 0 & 0 & 40 & 80 & 65 & 100 & 0 & 68 & 44,125 & 3 & 67 & $11 / 14 / 2006$ \\
\hline & $\mathrm{F}$ & 50 & 100 & 80 & 100 & 80 & 100 & 100 & 76 & 85,75 & & & \\
\hline
\end{tabular}




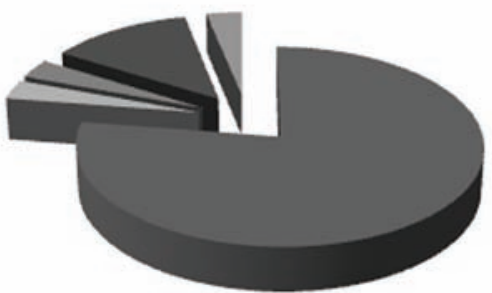

Doença degenerativa discal

Espondilolistese degenerativa

- Degeneração discal adjacente após artrodose

- Hernia discal recorrente

- Ruptura interna discal

\section{Figura 1}

Dignósticos mais comuns em pacientes submetidos ao tratamento cirúrgico.

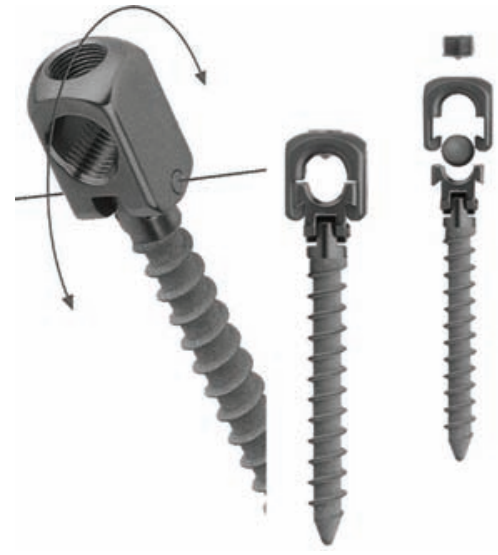

Figura 2

Parafusos do sistema cosmic-Ulrich (Reprodução de Stremple et al. ${ }^{16}$ ).
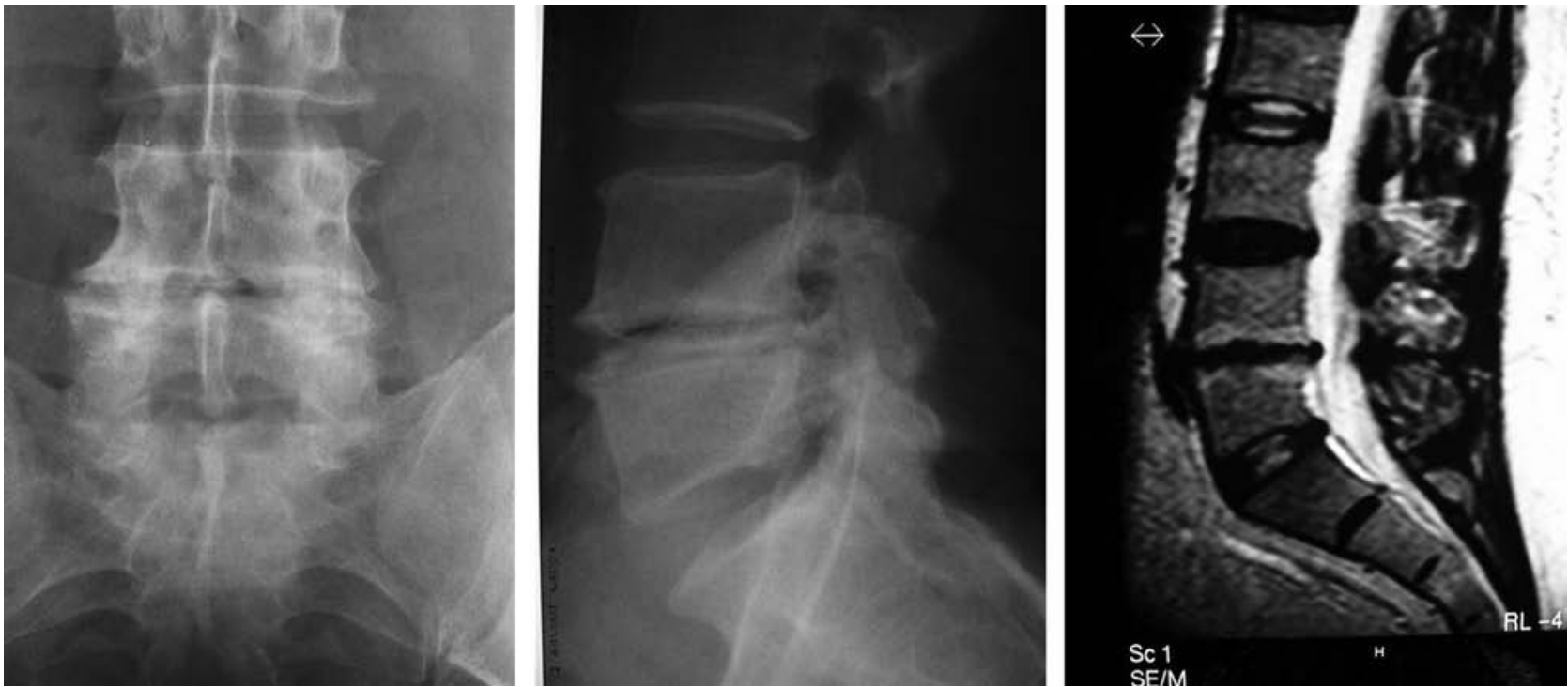

Figura 3

Paciente de 54 anos, sexo masculino, com quadro clínico de dor incapacitante em ortostase e à marcha. Exames mostram doença degenerativa discal com alteração mais acentuada no nível L4-L5.

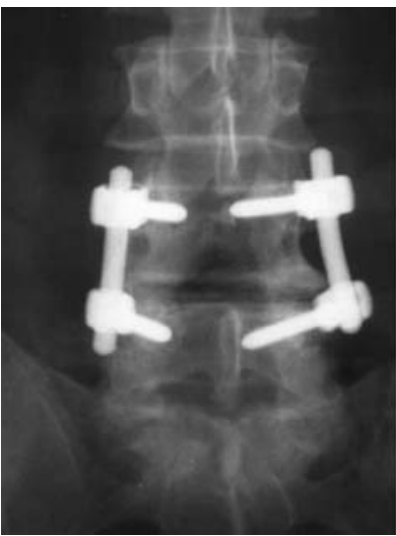

Figura 4

Radiografias simples nas incidências anteroposterior e perfil com quatro semanas de pós-operatório do paciente da Figura 3. Foi realizada laminectomia de $L 4$ com estabilição dinâmica pedicular do nível L4-L5.
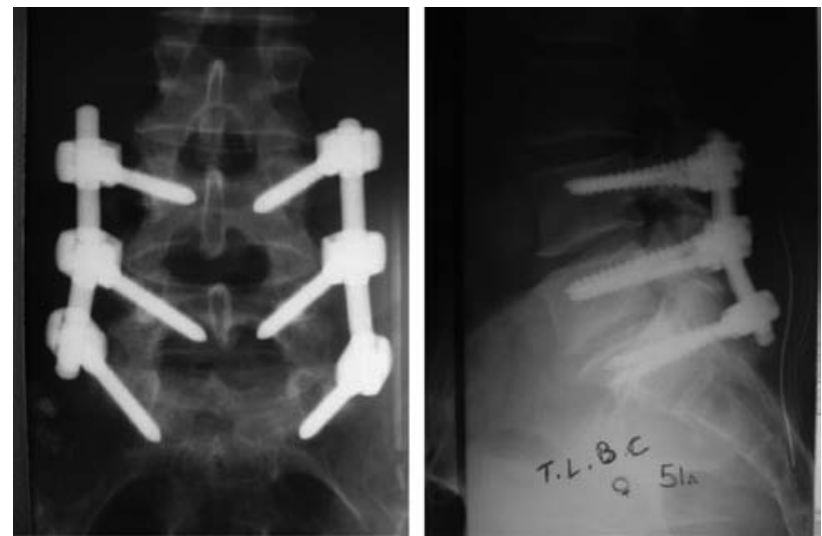

Figura 5

Exemplo de estabilização dinâmica em dois níveis, mostrando a convergência proposta para os parafusos. 
de entrada dos parafusos, que são colocados sob controle fluoroscópico ( $\operatorname{arco} \mathrm{em} \mathrm{C}$ ). Antibioticoterapia foi feita com cefazolina $2 \mathrm{~g}$ no pré-operatório, com nova dose de $1 \mathrm{~g}$ após três horas no intraoperatório ou em caso de perda sanguína maior do que 1 litro, mantendo-se cobertura antibiótica profilática com $1 \mathrm{~g}$ de cefazolina, de 8 em 8 horas, durante 24 horas no pós-operatório. Drenos a vácuo foram utilizados em todos os pacientes e retirados, em média, 48 horas após o término do procedimento. Tromboprofilaxia foi feita apenas após 24 horas, usando heparina de baixo peso molecular (enoxaparina $40 \mathrm{mg}$ subcutânea), até o momento da alta hospitalar. Os pacientes foram colocados em posição sentada no dia seguinte e iniciaram treino de marcha no máximo 48 horas após a cirurgia, para mobilidade precoce, que diminui o risco de complicações pulmonares e vasculares, além de melhorarem a recuperação funcional, juntamente com a fisioterapia motora e respiratória, que foi iniciada logo nas primeiras 24 horas do pós-operatório.

O questionário de avaliação de qualidade de vida utilizado foi o SF-36 (Short Form Health Survey). Tratase de um questionário multidimensional composto por 36 questões, que são base de cálculos para uma avaliação de oito componentes: (1) capacidade funcional, (2) aspectos físicos, (3) dor, (4) estado geral de saúde, (5) vitalidade, (6) aspectos sociais, (7) aspectos emocionais e (8) saúde mental.
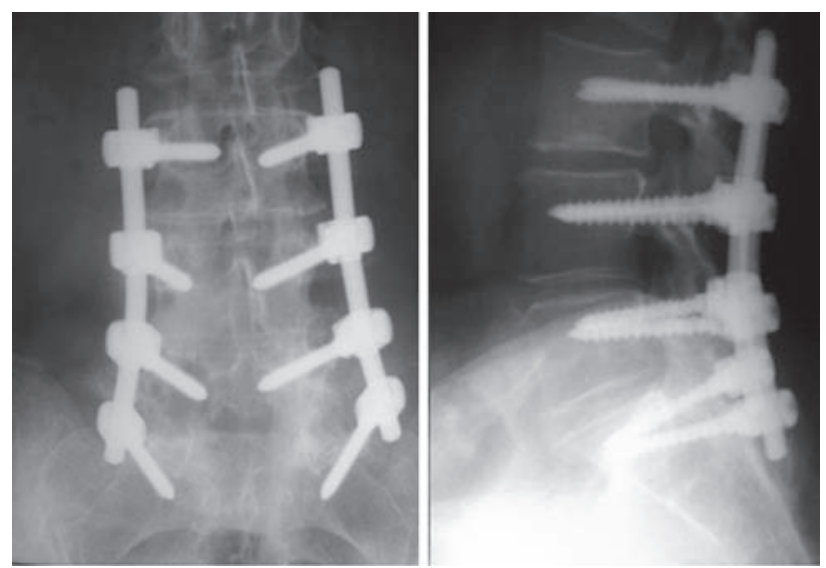

Figura 6

Exemplo de estabilização dinâmica pedicular em três níveis.

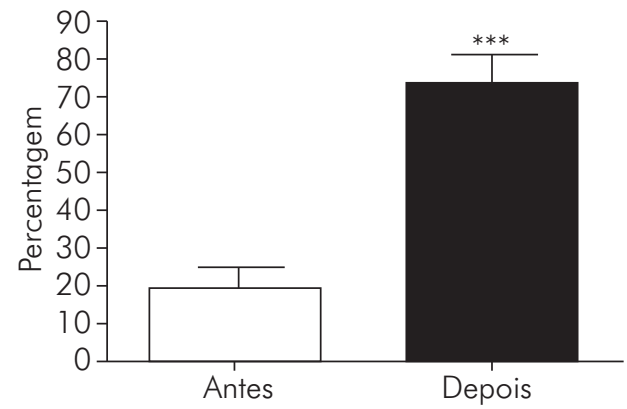

Gráfico 2

Limitação por aspectos físicos
A partir destes resultados, foi feita avaliação estatística pareada, com teste $t$ de Student, nos períodos em que o questionário de avaliação de qualidade de vida foi respondido (pré e pós-operatório).

No final, foi feita uma média geral dos resultados obtidos a fim de demonstrar a variação geral da média do questionário.

\section{RESULTADOS}

A capacidade funcional mostrou uma diferença de $26,45 \%$ (pré-operatório) para $80,12 \%$ (pós-operatório), com uma melhora de $202,9 \%$ ( $<<0,0001$ ), como mostrado no gráfico 1 .

Nas limitações por aspectos físicos, a diferença foi de $18,54 \%$ (pré-operatório) para 73,38\% (pós-operatório), com uma melhora de $295 \%$ ( $<<0,0001)$, como mostrado no gráfico 2 .

Em relação à dor, o escore variou de 29,38\% (pré-operatório) a $65,19 \%$ (pós-operatório), com uma melhora de $121,88 \%(\mathrm{p}<0,0001)$, como mostrado no gráfico 3.

O estado geral de saúde variou de $47,2 \%$ (pré-operatório) para 78,5\% (pós-operatório), com uma melhora de $66,3 \%$ ( $<<0,0001)$, como mostrado no gráfico 4 .

Em relação à vitalidade, a variação foi de $35,96 \%$ (préoperatório) a $67,09 \%$ (pós-operatório), com uma melhora de $86,56 \%$ ( $<<0,0001)$, como mostra o gráfico 5 .

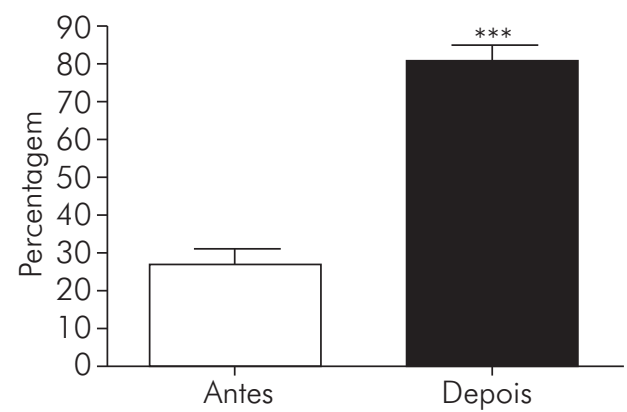

Gráfico 1

Capacidade funcional

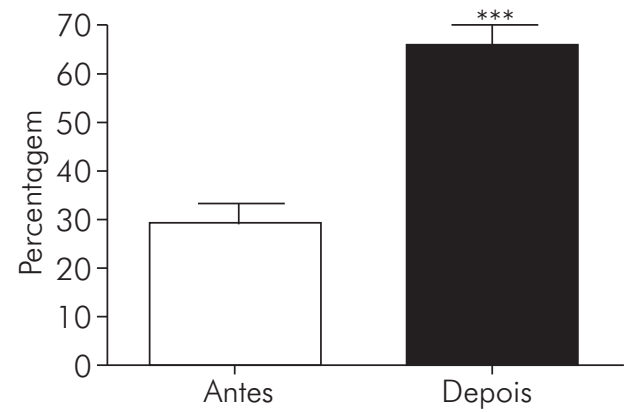

Gráfico 3

Dor 
$\mathrm{Na}$ limitação por aspectos sociais, a média variou de $37,5 \%$ (pré-operatório) para 80,64\% (pós-operatório), com uma melhora de $115 \%$ ( $<<0,0001)$, como mostrado no gráfico 6 .

$\mathrm{Na}$ limitação por aspectos emocionais, a média variou de $28,2 \%$ (pré-operatório) a 71,98\% (pós-operatório), com uma melhora de $155,24 \%$ ( $<<0,0001$ ), como mostrado no gráfico 7 .

Em relação à saúde mental, houve variação de 41,41\% (pré-operatório) para 74,96\% (pós-operatório), com uma melhora de $81 \%(\mathrm{p}<0,0001)$, como mostra o gráfico 8 .

$\mathrm{Na}$ média final, o índice SF-36 apresentou uma variação pré-operatória de $33,15 \%$ a $75,99 \%$ no pós-operatório, com uma melhora de $129,23 \%$, um resultado estatisticamente significativo $(p<0,0001)$ na melhora da qualidade de vida, como se observa no gráfico 9 .

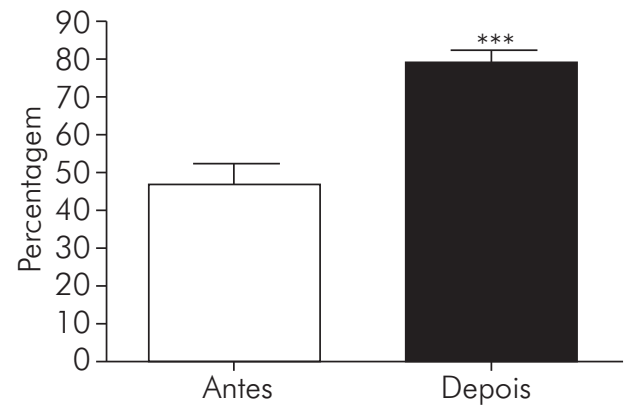

Gráfico 4

Estado geral de saúde

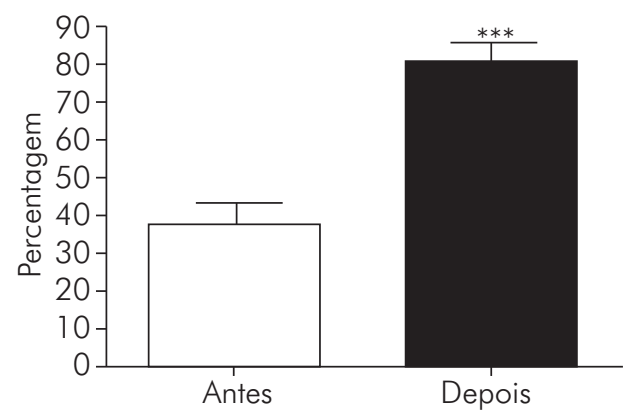

Gráfico 6

Aspectos sociais

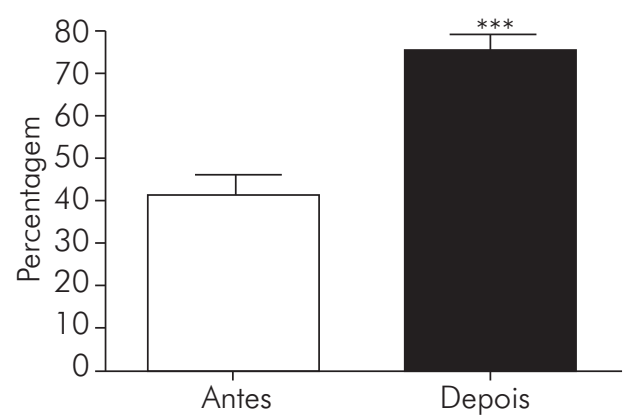

Gráfico 8

Saúde mental
Entre as complicações que ocorreram durante o período em que os pacientes foram acompanhados, pode-se mencionar: um paciente teve quebra de um dos parafusos durante o segundo ano de acompanhamento, havendo necessidade de revisão para a retirada do parafuso, com melhora do quadro álgico, e um paciente apresentou dor por degeneração muscular, obtendo melhora com medidas de fisioterapia. Não houve nenhum caso de infecção.

\section{DISCUSSÃO}

A indicação da técnica cirúrgica depende do tipo de patologia a ser tratada ${ }^{16}$. A fusão das unidades funcionais degeneradas é a técnica cirúrgica mais comumente indicada para estas patologias, e apresenta resultados satisfatórios para as dores lombares discogênicas ${ }^{17}$. Christensen demonstrou, em 2004, 70\% de bons resultados com artrodese a longo prazo ${ }^{6}$.

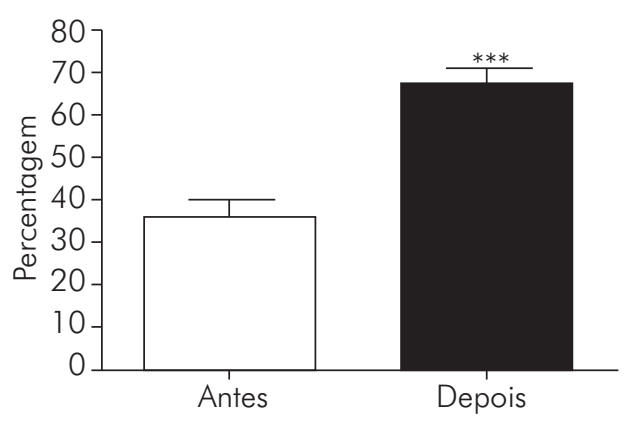

Gráfico 5

Vitalidade

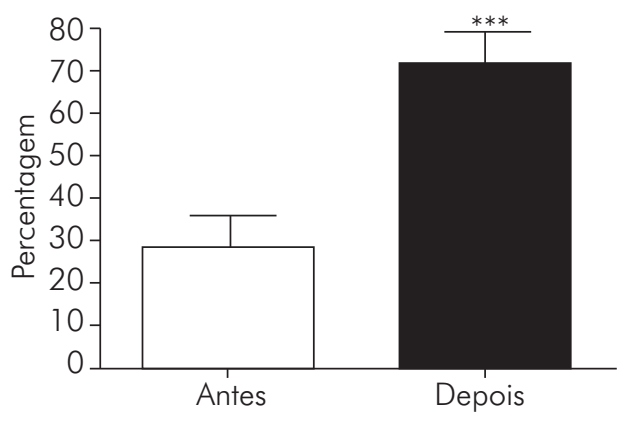

Gráfico 7

Limitação por aspectos emocionais

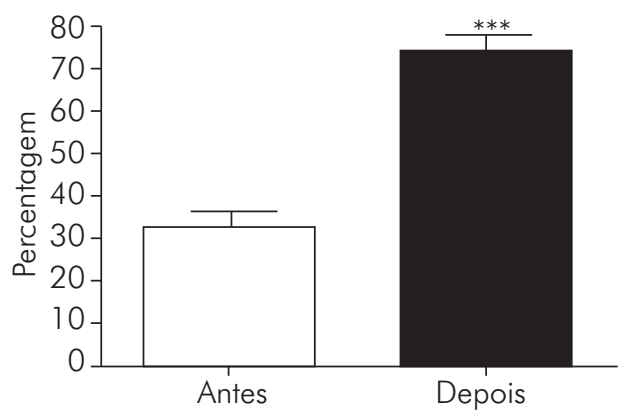

Gráfico 9

Média SF-36 
Os resultados de artroplastia lombar apresentados por Delamarter et al. ${ }^{18}$, no ano de 2003, em estudo comparativo com artrodese, mostram que, após seis semanas, as artroplastias apresentam melhora significativa nos índices de avaliação. O mesmo estudo mostra que, após seis meses, os resultados comparativos de artroplastia e artrodese são semelhantes, sem diferenças significativas.

A técnica cirúrgica de estabilização pedicular dinâmica é bastante simples e pouco diferente das fixações pediculares para fusão lombar. As indicações cirúrgicas são equivalentes para as patologias, às quais frequentemente se indicaria artrodese $\mathrm{e}^{16}$.

Os critérios de inclusão e exclusão devem ser respeitados para a seleção do paciente a fim de diminuir os riscos de complicações. A estabilização dinâmica é uma técnica cirúrgica com menor morbidade per-operatória que a fusão, já que não requer decorticação facetária ou retirada de enxerto, procedimentos necessários para artrodese (fusão $)^{16}$. Portanto, diminui o índice de complicações, propiciando um tempo cirúrgico mais curto, com menor sangramento e, como não requer retirada de enxerto, não há complicações relativas ao sítio doador. As técnicas de nãofusão não dependem de consolidação óssea, o que é uma vantagem para a recuperação pós-operatória, dependendo apenas da integração óssea da prótese ao corpo vertebral ${ }^{16}$.

O período médio de acompanhamento foi de 29,5 meses. Neste período, houve uma complicação de quebra do parafuso e da haste em um paciente que evoluiu com dor após dois anos com o implante. Neste caso, optamos pela revisão cirúrgica para a retirada do material, fato que causou a melhora do quadro álgico do paciente. Observou-se que o fato de os parafusos serem revestidos em hidróxiapatita dificulta a retirada do implante caso necessite de revisão, devido à grande osteointegração.

A técnica proposta por Strempel et al. não restringe atividades físicas e todos os esportes, sem restrições, são incentivados. Até o momento, não houve na presente série complicações com os níveis adjacentes à cirurgia.

Dois outros trabalhos da literatura que avaliaram os resultados obtidos em pacientes submetidos a procedimentos cirurgicos com o mesmo sistema dinâmico utilizado neste estudo (sistema Cosmic-Ulrich) foram publicados em 2006 e 2008, ambos por Stremple et al. ${ }^{16,19}$. O primeiro avaliou prospectivamente 96 pacientes, num período de 24 meses, utilizando a escala visual analógica de dor (VAS) e o Oswestry Low Back Pain Scale. O segundo trabalho é um estudo multicêntrico europeu, também prospectivo, envolvendo já um grupo maior, de 139 pacientes, também em um tempo de seguimento de 24 meses, e usando os mesmos parâmetros (questionários) da avaliação feita em 2006.

Em nosso estudo, 31 pacientes foram retrospectivamente avaliados num período de 7 a 57 meses, utilizando como método de avaliação clínica o questionário de qualidade de vida SF-36.

O grupo do Stremple observou um índice de $1,04 \%$ de infecção de ferida cirúrgica em seu estudo de 2006 e de 3,6\% em 2008. Dentre as complicações apresentadas, no presente estudo não houve casos de infecção de ferida cirúrgica.

$O$ Loosening do parafuso ocorreu em 5,2\% dos parafusos no primeiro estudo realizado pelo grupo do Stremple, e em 5,03\% em seu segundo estudo. Em comparação, não há nenhum caso na presente série.

Complicações como degeneração de discos adjacentes, hematoma pós-cirúrgico e fratura de vértebra cranial da instrumentação devido à osteoporose, e que necessitaram de revisão, só foram observadas no estudo multicêntrico europeu (segundo estudo do grupo de Stremple), sendo que cada uma destas ocorreu em apenas um caso $(0,7 \%)$.

A única complicação do método que se observou em todos os estudos aqui comparados foi a quebra dos parafusos, sendo 2,08\% no primeiro estudo do grupo do Stremple em 2006, 1,43\% no estudo multicêntrico europeu de 2008, e $3,22 \%$ na presente amostra. Porém, vale ressaltar que nem todas as quebras foram sintomáticas, sendo que a taxa de revisão mostrada nestes estudos relacionadas à quebra dos parafusos foi inferior à taxa de quebra dos parafusos.

Em relação aos resultados clínicos, avaliados pelos questionários acima descritos, o primeiro trabalho do grupo do Stremple mostrou melhora do Oswestry de 50,8 a $34 \%$, e na VAS de 5,7 de $2,9 \%$. O estudo multicêntrico europeu mostrou melhora do índice do Oswestry de 48,7 para $20,6 \%$, e na VAS de 7,1 para 2,7\%. Neste estudo, apenas se utilizou o método de avaliação do questionário SF-36, mostrando uma melhora de 33,15 para $75,99 \%(p<0,0001)$ na qualidade de vida dos pacientes entre os períodos pré e pós-operatório.

Estudos de estabilização dinâmica desenvolvidos por Grob et al. ${ }^{20}$ no ano de 2005, utilizando o sistema Dynesys, também de parafusos pediculares, associados a uma haste de polyester e a um espaçador de policarbonato uretano, apresentam bons resultados, porém com alto índice de revisão cirúrgica.

\section{CONCLUSÃO}

As doenças degenerativas discais lombares, quando não diagnosticadas corretamente antes do procedimento cirúrgico, comprometem o resultado final do tratamento. A utilização da estabilização dinâmica como técnica cirúrgica é recente. Os resultados ainda são preliminares e as publicações são escassas. A técnica com parafusos pediculares em sistema dinâmico ainda está sendo estudada em diversos centros. Os resultados deste estudo com o sistema cosmic-Ulrich de estabilização dinâmica são satisfatórios nesta avaliação parcial, mostrando diferença estatisticamente significativa na melhora do questionário de qualidade de vida SF-36 destes pacientes no que diz respeito aos períodos pré e pós-operatórios.

\section{AGRADECIMENTOS}

Agradecemos aos pacientes que colaboraram com este estudo, ao responder aos questionários, e à Tania Spohr, pela cuidadosa revisão do artigo. 


\section{REFERÊNCIAS}

1. White AA III, Panjabi MM. Clinical biomechanics of the spine. 2nd ed. Philadelphia: J.B. Lippincott Co.; 1990. Biomechanics A to Z. p. 635-96.

2. Kruton F. The instability associated with disc degeneration in the lumbar spine. Acta-Radio. 1944;25:593-609.

3. Morgan FP, Krug T. Primary instability of lumbar vertebrae as common cause of low back pain. J Bone Joint Surg. 1957;39(1):6-22.

4. Kirkaldy-Willis WH, Wedge JH, Yong-Hing K, Reilly J. Pathology and pathogenesis of lumbar spondylosis and stenosis. Spine. 1978;3(4):319-28.

5. Kirkaldy-Willis WH, Farfan HF. Instability of the lumbar spine. Clin Orthop Relat Res. 1982(185):110-23.

6. Christensen FB. Lumbar spinal fusion. Outcome in relation to surgical methods, choice of implants and postoperative rehabilitation. Acta Orthop Scand. 2004;75(313):2-43.

7. Louis R. Spinal stability as defined by the three-column spine concept. Anat Clin. 1985;7(1):33-42.

8. Kazarian LE. Creep characteristics of the human spinal column. Orthop Clin North Am. 1975;6(1):3-18.

9. Lumsden RM, Morris JM. An in vivo study of axial rotation and immobilization at lumbosacral joint. J Bone Joint Surg. 1968;50(8):1591-602.

10.Rahm MD, Hall BB. Adjacentsegment degeneration after lumbar fusion with instrumentation: a retrospective study. J Spinal Dis. 1996;9(5):392-400.
11.Luk KD, Lee FB, Leong JC, Hsu LC. The effect on the lumbosacral spine of long spinal fusion for idiopathic scoliosis. A minimum 10-year followup. Spine. 1987;12(10):996-1000.

12.Kornblum MB, Fischgrund JS, Herkowitz HN, Abraham DA, Berkower DL, Ditkoff JS. Degenerative lumbar spondylolisthesis with spinal stenosis: a prospective long-term study comparing fusion and pseudarthrosis. Spine. 2004;29(7):726-33.

13.Buttermann GR, Garvey TA, Hunt AF, Transfeldt EE, Bradford DS, Boachie-Adjei O, et al. Lumbar fusion results related to diagnosis. Spine. 1998;23(1):116-27.

14.Mulholand RC, Sengupta DK. Rationale, principles and experimental evaluation of the concept of soft stabilization. Eur Spine J. 2002;11 Suppl 2:S198-205.

15.Ghiselli G, Wang JC, Bhatia NN, Hsu WK, Dawson EG. Adjacent segment degeneration in the lumbar spine. J Bone Joint Surg Am. 2004;86(7):1497-503.

16. Strempel A, Moosmann D, Stoss C, Martin A. Stabilization of the degenerated lumbar spine in the non fusion technique with Cosmic posterior dynamic system. World Spine J. 2006;1(1):40-7.
17.Ragab A, Flanum M, Galanis C, Zdeblick T. Anterior lumbar interbody fusion for treatment of low back pain internal disc disruption versus degenerative disc disease. Spine J. 2002;2(5):102.

18.Delamarter RB, Fribourg DM, Kanin LE, Bae H. ProDisc artificial total lumbar disc replacement: introduction and early results from the United States clinical trial. Spine. 2003;28(20):167-75.

19. Stremple A. Dynamic stabilisation: cosmic system. Interact Surg. 2008,3(4):229-36.

20.Grob D, Benini A, Junge A, Mannion AF. Clinical experience with the Dynesis semirigid fixation system for the lumbar spine: surgical and patient-oriented outcome in 50 cases after an average of 2 years. Spine. 2005;30(3):324-31.

\section{Correspondência:}

Leonardo Fonseca Rodrigues

Centro Ortopédico da Barra

Avenida das Américas, 679, CoberturaBarra da Tijuca

CEP: 22640-102 - Rio de Janeiro (RJ), Brasil

E-mail: leonardofrodrigues@gmail.com 